\title{
Cantilever Tilt Causing Amplitude Related Convolution in Dynamic Mode Atomic Force Microscopy
}

\author{
Chunmei WANG, ${ }^{* 1}$ Jielin Sun, ${ }^{* 1 \dagger}$ Hiroshi ITOH, ${ }^{* 2}$ Dianhong SHEN, ${ }^{* 3}$ and Jun HU ${ }^{* 4}$ \\ *1 School of Life Science and Biotechnology \& Ministry of Education, Key Laboratory for Systems Biomedicine, \\ Shanghai Jiaotong University, 800 Dongchuan Road, Shanghai 200240, P. R. China \\ *2 Research Institute of Instrumentation Frontier, National Institute of Advanced Industrial Science and \\ Technology (AIST), 1-1-1 Umezono, Tsukuba, Ibaraki 305-8568, Japan \\ *3 National Center for Nanoscience and Nanotechnology, No. 2 ZhongGuanCun BeiYiJie, Beijing 100080, \\ P. R. China \\ *4 Shanghai Institute of Applied Physics, Chinese Academy of Sciences, 2019 Jialuo Road, Jiading District, \\ Shanghai 201800, P. R. China
}

\begin{abstract}
It is well known that the topography in atomic force microscopy (AFM) is a convolution of the tip's shape and the sample's geometry. The classical convolution model was established in contact mode assuming a static probe, but it is no longer valid in dynamic mode AFM. It is still not well understood whether or how the vibration of the probe in dynamic mode affects the convolution. Such ignorance complicates the interpretation of the topography. Here we propose a convolution model for dynamic mode by taking into account the typical design of the cantilever tilt in AFMs, which leads to a different convolution from that in contact mode. Our model indicates that the cantilever tilt results in a dynamic convolution affected by the absolute value of the amplitude, especially in the case that corresponding contact convolution has sharp edges beyond certain angle. The effect was experimentally demonstrated by a perpendicular $\mathrm{SiO}_{2} / \mathrm{Si}$ super-lattice structure. Our model is useful for quantitative characterizations in dynamic mode, especially in probe characterization and critical dimension measurements.
\end{abstract}

(Received August 17, 2010; Accepted November 24, 2010; Published February 10, 2011)

\section{Introduction}

Atomic force microscopy (AFM), one of many examples of scanning probe microscopy (SPM), is a powerful technique in nanoscale. ${ }^{1}$ It exhibits the unique capabilities of imaging a wide range of nanostructures in various environments and has superior resolution down to atomic scale and single molecule level. ${ }^{1,2}$ With the maturity of SPM techniques, SPM quantitative analysis and standardization have become an urgent task in the community of nanoscience and nanotechnology.,4 The increasing demands of reproducibility and precision in SPM measurements are triggering not only the advancement of the reference specimens, but also the studies on the modeling for achieving more accurate measurements and analyses.

Convolution, the well-known artifact in SPM images which leads to the difficulties in quantitative measurements, ${ }^{5,6}$ is now one of the essential topics in SPM standardization. ${ }^{3,4}$ The standard specimen, the theoretical convolution/deconvolution model and the standardized characterization process, are necessary for quantitatively analyzing and reducing this effect. Recently, the dimension of the tip characterizer has been greatly improved by the successful development of a new kind of tip characterizer specimen based on superlattice techniques.?

$\dagger$ To whom correspondence should be addressed.

E-mail: jlsun@sjtu.edu.cn
However, the more practical and optimized characterization procedures in various AFM imaging modes are required with the related studies on the model's applicability, experimental optimization and error analysis. It should be noted that the conventional convolution model, one of the base for SPM quantitative analysis, was established based on contact mode, and has been directly referred in dynamic mode. The introduction has always been thought to be reasonable since it is taken for granted that the cantilever is mounted horizontally to the sample plane and then the probe vibrates vertically. However, the cantilever is universally designed in the practical instruments with a $7-20^{\circ}$ tilt instead of the horizontal case, ${ }^{8}$ and some significant corrections of several models resulted from the cantilever tilt have been reported and emphasized. ${ }^{8-12}$ Since the cantilever tilt obviously leads to a different tilted vibrating probe in dynamic mode, one has to address whether the convolution in dynamic mode is really the same as that in contact mode or not before seeking more precise and reproducible analyses in dynamic mode AFM.

In this paper, we reinvestigated the convolution model in dynamic mode AFM with a tilted cantilever. We found that the tilted vibration of the probe will affect the convoluted topography. With the peak-peak vibration amplitude $(2 A)$ and the tilted vibration direction, related to cantilever tilt angle $\alpha$, involved as the length and the direction of an additionally imaginary line probe, a new convolution model for dynamic mode was established by convoluting the corresponding 
(a) contact convolution

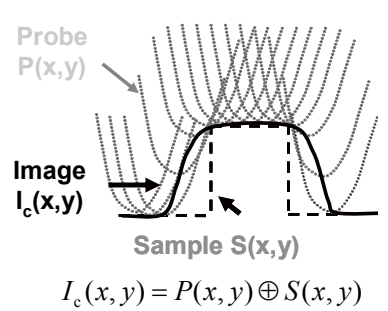

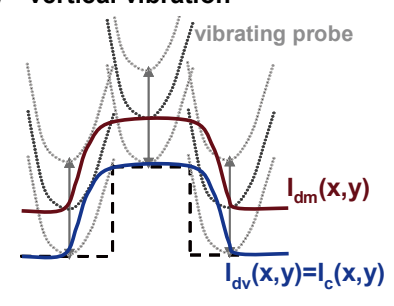

$$
I_{\mathrm{dm}}(x, y)=I_{\mathrm{dv}}(x, y)+A
$$

(b) vertical vibration

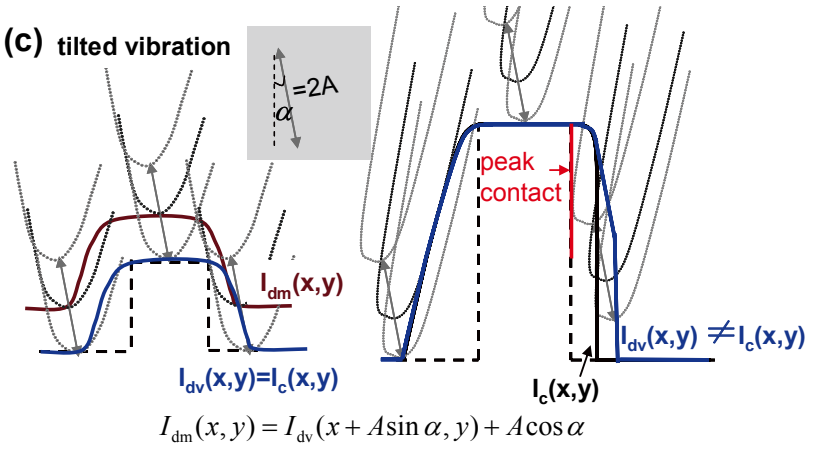

Fig. 1 The different convolution processes in contact mode and in dynamic mode. (a) The conventional convolution $I_{\mathrm{c}}(x, y)$ from the contact state. (b) The dynamic convolution $I_{\mathrm{dm}}(x, y)$ from the midpoint of one oscillation. For the vertical vibration case, i.e., the cantilever is horizontal, the trajectory $I_{\mathrm{dv}}(x, y)$ from the valley-point of the oscillation has $I_{\mathrm{dv}}(x, y)=I_{\mathrm{c}}(x, y)$. (c) The dynamic convolution in the case of the tilted vibration, i.e., the cantilever is tilted. $I_{\mathrm{dv}}(x, y)=I_{\mathrm{c}}(x, y)$ when only the valley contacts exist (left), and $I_{\mathrm{dv}}(x, y) \neq I_{\mathrm{c}}(x, y)$ when the peak contacts happen (right). $I_{\mathrm{dm}}(x, y)$ and corresponding $I_{\mathrm{dv}}(x, y)$ have the same shape.

contact convoluted topography with the line probe. The model indicates that, if the corresponding contact convoluted topography has edges with the surface angles sharper than $\left(\alpha-90^{\circ}\right)$, the dynamic topography is proportionally expanded relative to the contact one with the increasing amplitude $A$. Using the designed specimen including $\mathrm{SiO}_{2} / \mathrm{Si}$ super-lattice structures with edge angles larger than 89 degrees and the high aspect ratio probe, an amplitude related effect on topographies in dynamic mode was demonstrated. Our model is especially valuable for accurate and quantitative analysis of sharp nanostructures in dynamic mode AFM, such as the tip characterizer and the specimens in critical dimension (CD) measurements.

\section{Theoretical Modeling and Calculations}

Basic convolution processes in contact mode and in dynamic mode

In the traditional convolution model established in contact mode, the convoluted topography $I_{\mathrm{c}}(x, y)$ is the apex's positions when the static probe contacts the sample ${ }^{5}$ (Fig. 1(a)). However, as shown in Fig. 1(b), the topography $I_{\mathrm{dm}}(x, y)$ in dynamic mode corresponds to the apex's positions at the mid-point of one oscillation, which is not the state when the probe contacts the sample. In the case that the probe vibrates vertically, the probe always contacts the sample at the valley-point of one oscillation, and the valley topography $I_{\mathrm{dv}}(x, y)$ is just equal to $I_{\mathrm{c}}(x, y)$. Since $I_{\mathrm{dm}}(x, y)$ only shifts in $z$ direction by the vibration amplitude $A$ relative to $I_{\mathrm{dv}}(x, y)$, there is no difference in the topographical shape between $I_{\mathrm{dm}}(x, y)$ and $I_{\mathrm{dv}}(x, y)$. As a result, the traditional convolution model can be reasonably applied (Fig. 1(b)).

However, due to the general design of the cantilever tilt in commercial AFMs, the probe in reality vibrates along a tilt direction in dynamic mode (Fig. 1(c)), which additionally leads to a lateral vibration component and therefore the probe becomes sensitive to the lateral variations in the structures. In this case, the mid-point topography $I_{\mathrm{dm}}(x, y)$ of the oscillation still has the same shape as the valley topography $I_{\mathrm{dv}}(x, y)$, but shifts in both $x$ and $z$ directions relative to $I_{\mathrm{dv}}(x, y)$ as follows:

$$
I_{\mathrm{dm}}(x, y)=I_{\mathrm{dv}}(x+A \sin \alpha, y)+A \cos \alpha
$$

If the probe-sample's contact state in this case is still the same as what is shown in the vertical vibration case (Fig. 1(b)) that the probe contacts the sample at the valley-point of one oscillation, the traditional convolution model is still applicable to $I_{\mathrm{dv}}(x, y)$ (Fig. 1(c), left). However, the illustration also shows the case that the probe is possible to contact the specimen at the peak-point of the oscillation, and then $I_{\mathrm{dv}}(x, y)$ gives an inconsistent result from the conventional convolution $I_{\mathrm{c}}(x, y)$ (Fig. 1(c), right). Therefore, it is necessary to establish a convolution model suitable for dynamic mode.

\section{Establish the convolution model for dynamic mode}

The convolution model for dynamic mode was established through the following steps:

i. Based on the definition in the contact convolution model, ${ }^{5}$ convolution between a finite size probe $P(x, y)$ and a specimen $S(x, y)$ can be equivalent to a point probe with radius $r \rightarrow 0$ scanning over the contact convoluted topography $I_{\mathrm{c}}(x, y)$.

ii. Then, the convolution of a vibrating probe with the shape $P(x, y)$ scanning over a specimen with the geometry $S(x, y)$ is equivalent to that of a vibrating point probe scanning over $I_{\mathrm{c}}(x, y)$ (Fig. 2(a)). With the peak-peak vibration amplitude $2 A$ and the direction $\alpha$ degree deviated from $z$ axis as the finite length and the direction of an imaginary line probe $P_{\mathrm{dt}}(x, y)$, the complex dynamic convolution equals the result that $P_{\mathrm{dt}}(x, y)$ convolutes with $I_{\mathrm{c}}(x, y)$ :

$$
\begin{aligned}
I_{\mathrm{dv}}(x, y) & =P_{\mathrm{dt}}(x, y) \oplus I_{\mathrm{c}}(x, y) \\
& =P_{\mathrm{dt}}(x, y) \oplus P(x, y) \oplus S(x, y)
\end{aligned}
$$

Equation (2) expressly shows that the vibration affects the dynamic convolution by adding an vibration-related item $P_{\mathrm{dt}}(x, y)$ into the conventional convolution model.

Let us suppose that $\beta(x, y)=\tan ^{-1}\left(\partial I_{\mathrm{c}}(x, y) / \partial x\right)\left(-90^{\circ}<\beta \leq 90^{\circ}\right)$ is the surface angle at any position $(x, y)$ on $I_{\mathrm{c}}(x, y)$. According to the definition of the cantilever-specimen system in this paper, it is easy to deduce geometrically from Fig. 2(b) that the probe contacts where $\beta>\alpha-90^{\circ}$ on $I_{c}(x, y)$ at the oscillation valley and contacts where $\beta<\alpha-90^{\circ}$ at the oscillation peak. If all $\beta$ over $I_{\mathrm{c}}(x, y)$ are larger than $\left(\alpha-90^{\circ}\right), I_{\mathrm{dv}}(x, y)$ equals $I_{\mathrm{c}}(x, y)$, as is the case shown in Fig. 1(c) left. Otherwise, there are mixed valley and peak contacts in the same topography, leading to the distinct right-side-only disagreement between $I_{\mathrm{dv}}(x, y)$ and $I_{\mathrm{c}}(x, y)$, and the $P_{\mathrm{dt}}(x, y)$ induced effect has to be considered in this case (Fig. 1(c) right and Fig. 2(b)). The phenomenon usually happens while measuring the sharp structure by the high aspect ratio probe such as out-growing CNT probes or similar special probes (Fig. 2(b), right). A deconvoluted surface $R_{\mathrm{c}}(x, y)$ with a reduced vibration influence, which is closer to $I_{\mathrm{c}}(x, y)$, can be recovered in this case from Eq. (3) if $A$ and $\alpha$ for $P_{\mathrm{dt}}(x, y)$ is accurately known: 


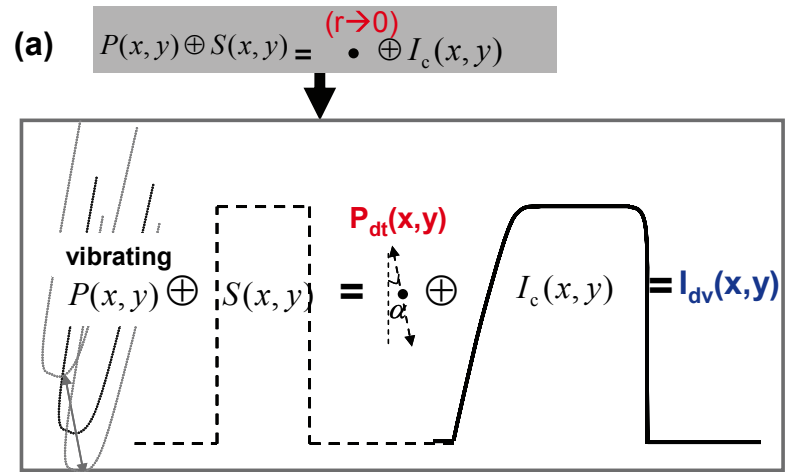

(b)

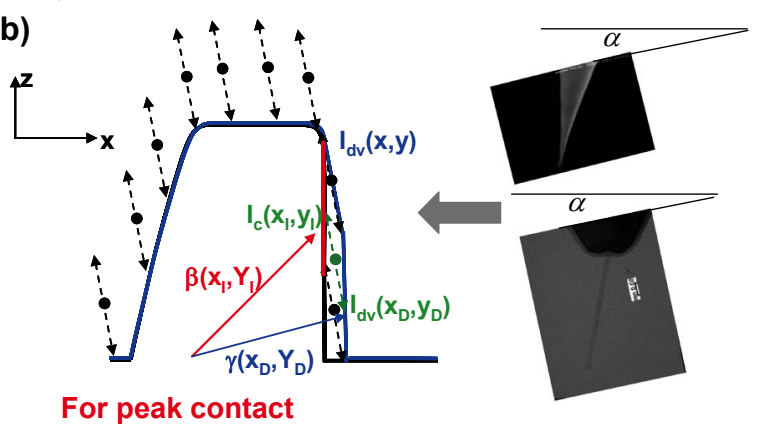

surface angle $\gamma=\beta<a-90^{\circ}$

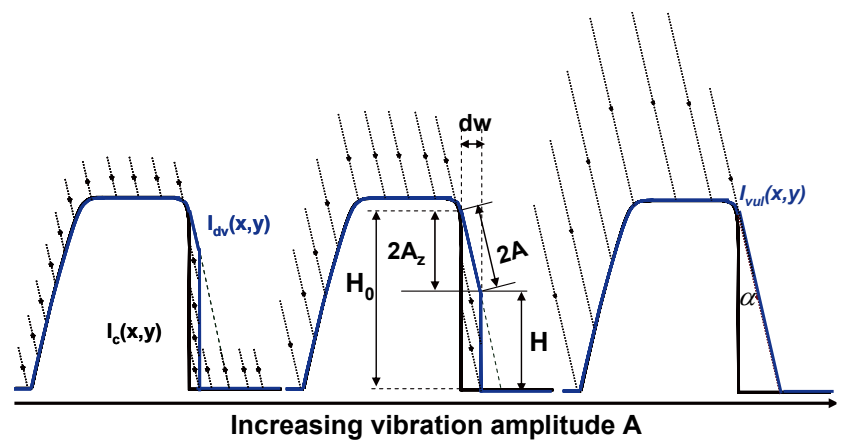

Fig. 3 The amplitude related effect on the convolution induced by the cantilever tilt. The increasing amplitude proportionally expands the dynamic convolution $I_{\mathrm{dv}}(x, y)$ in $x-z$ plane. (a)

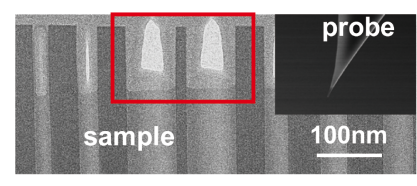

(b)
Fig. 2 The convolution model for dynamic mode with the cantilever tilt. (a) The establishment of the dynamic convolution model. $I_{\mathrm{dv}}(x, y)$ equals the convolution result between $I_{\mathrm{c}}(x, y)$ and an imaginary line probe $P_{\mathrm{dt}}(x, y)$, with the finite length $2 A$ along the vibration direction. (b) The condition that $P_{\mathrm{dt}}(x, y)$ has a real effect on the convolution result. Where the surface angle $\beta>\left(\alpha-90^{\circ}\right)$ has the valley contact, and where $\beta<\left(\alpha-90^{\circ}\right)$ has the peak contact. The peak contact contains a $P_{\mathrm{dt}}(x, y)$ effect and can be judged experimentally from whether there is the surface angle $\gamma$ on $I_{\mathrm{dv}}(x, y)$ smaller than $\left(\alpha-90^{\circ}\right)$ Some special probes can invoke the effect (right).

$$
R_{\mathrm{c}}(x, y)=I_{\mathrm{dv}}(x, y) \ominus P_{\mathrm{dt}}(x, y)
$$

Then, the structure can be further deconvoluted to reduce the effect from the actual probe shape, as is done in the conventional convolution model.

In Fig. 2(b), suppose $I_{\mathrm{c}}\left(x_{\mathrm{I}}, y_{\mathrm{I}}\right)$ with the surface angle $\beta\left(x_{\mathrm{I}}, y_{\mathrm{I}}\right)<$ $\alpha-90^{\circ}$ is a peak contact position on contact $I_{\mathrm{c}}(x, y)$ and $I_{\mathrm{dv}}\left(x_{\mathrm{D}}, y_{\mathrm{D}}\right)$ is the corresponding valley position included in dynamic $I_{\mathrm{dv}}(x, y)$. Distinctly, the surface angle $\gamma\left(x_{\mathrm{D}}, y_{\mathrm{D}}\right)$ at $I_{\mathrm{dv}}\left(x_{\mathrm{D}}, y_{\mathrm{D}}\right)$ equals to $\beta\left(x_{\mathrm{I}}, y_{\mathrm{I}}\right)<\alpha-90^{\circ}$. This indicates that wherever $\gamma(x, y)<\alpha-90^{\circ}$ on $I_{\mathrm{dv}}(x, y)$ corresponds to the peak contact state, and it can be used to discriminate whether the vibration effect is included in the dynamic topography.

\section{Imaging amplitude effect in dynamic convolution}

Figure 2(b) indicates that the length of the line probe $P_{\mathrm{dt}}(x, y)$, that is, the vibration amplitude $A$, is closely related to the difference between dynamic $I_{\mathrm{dv}}(x, y)$ and contact $I_{\mathrm{c}}(x, y)$. Distinctly, $I_{\mathrm{dv}}(x, y)$ tends to $I_{\mathrm{c}}(x, y)$ for $A \rightarrow 0$. In the typical case that $A \neq 0, I_{\mathrm{dv}}(x, y)$ is expanded at the right side with increasing $A$ along a tilted line with the vibration direction (dotted line, Fig. 3), resulting in synchronously increasing difference between $I_{\mathrm{dv}}(x, y)$ and $I_{\mathrm{c}}(x, y)$ (left $\rightarrow$ right, Fig. 3). The expanded topography reaches an up-limit $I_{\mathrm{vul}}(x, y)$ after $A$ increases to a threshold amplitude (right, Fig. 3).

In the simple but common situation shown in Fig. 3, define $H$ as the height over the region with $\gamma<\alpha-90^{\circ}$ on dynamic
Fig. 4 (a) The SEM image of the probe with the same type as used in the experiment (inset) and a newly developed 2D structure made by $\mathrm{SiO}_{2} / \mathrm{Si}$ superlattice with perpendicular edges. (b) AFM image (inset) of the isolated line enclosed in (a) and the line profiles (trace and retrace).

$I_{\mathrm{dv}}(x, y)$ and $H_{0}$ as the height over the region with $\beta<\alpha-90^{\circ}$ on contact $I_{\mathrm{c}}(x, y)$. Then the width $d_{\mathrm{w}}$ expanded by the vibration amplitude is estimated as:

$$
d_{\mathrm{w}}=2 A \sin \alpha=\left(H_{0}-H\right) \tan \alpha
$$

The recoverable peak contact region $H$ is:

$$
H=H_{0}-2 A \cos \alpha=H_{0}-2 A_{\mathrm{z}}
$$

Here $A_{\mathrm{z}}$ is the vertical projection of $A$.

It has to be noted that the above analyses for dynamic convolution are based on the assumption of both a rigid sample and no long range forces, as usually supposed in contact convolution model. However, in most experiments, the sample might have deformation and other physical/chemical properties. Thus, the situation becomes more complex. Even so, the criterion for peak/valley contact is applicable in principle, and we might get a reconstructed "effective contact" surface $R_{\mathrm{c}}(x, y)$ for further analyses.

\section{Experimental}

The experiments were designed and conducted to verify the special amplitude effect on topographies deduced from the established dynamic convolution model. All experiments were conducted in dynamic mode in air by using a commercial AFM manufactured by Veeco Inc. The relative humidity $(\mathrm{RH})$ was maintained at $\sim 30 \%$ and the temperature was near $25^{\circ} \mathrm{C}$. A high aspect ratio silicon probe with spring constant $1.2-3.5 \mathrm{~N} / \mathrm{m}$ 

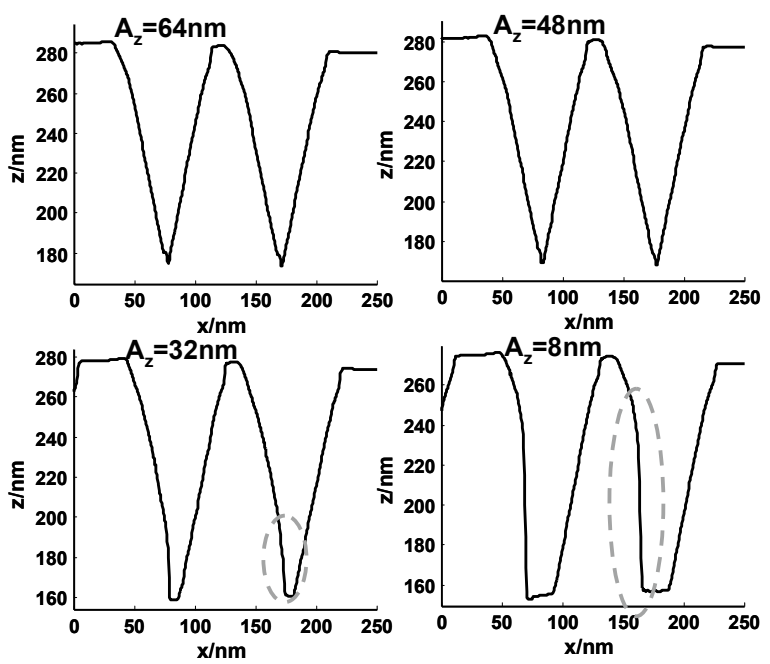

Fig. 5 The change of the experimental profile with the decreasing amplitude: the topography became sharper and sharper after $A_{\mathrm{z}}$ was decreased to the threshold value $48 \mathrm{~nm}$.

(Fig. 4(a) inset) and a well-defined 2D sample with perpendicular edges made from $\mathrm{SiO}_{2} / \mathrm{Si}$ superlattices (Fig. 4(a)) were used to successfully observe the cantilever tilt-induced effect. The isolated line structure enclosed on the TEM image by the red box in Fig. 4(a) was selected for AFM imaging (Fig. 4(b)) and further analysis.

The free amplitude $A O_{\mathrm{z}}$ was chosen to be around $80 \mathrm{~nm}$, which guaranteed enough repulsive force regimes for imaging while adjusting the imaging amplitude $A_{\mathrm{z}}$. Then the isolated line structure was measured by first decreasing (partial data shown in Fig. 5) and then reversely increasing (data not shown) the amplitude $A_{z}$. The decreasing/increasing step size of the imaging amplitude was set as $8 \mathrm{~nm}$. The parameters were carefully adjusted to make trace and retrace phase signals overlap, which minimized the differences between the trace and retrace topographies ${ }^{13}$ (Fig. 4(b)). After finishing all topographical measurements, we measured the amplitude-distance curve to convert the unit of the amplitude from voltage to nanometer, according to the fact that its slope should be equal to 1 . The converted amplitude value by this method is for $A_{z}$.

\section{Results and Discussion}

With the decrease of the imaging amplitude $A_{z}$, the line profiles of the isolated line structure endured no change in the case that $A_{z}$ was larger than $48 \mathrm{~nm}$. However, after $A_{\mathrm{z}}$ was decreased to a value less than $48 \mathrm{~nm}$, the right side of the line profile shrank to smaller and smaller value with continuously decreasing $A_{\mathrm{z}}$ (Fig. 5), as was discussed and deduced in Fig. 3. It was a reversible process with increasing $A_{\mathrm{z}}$ (not shown).

All line profiles under different $A_{\mathrm{z}}$, after removing the lateral and vertical drift by using normalized cross-correlation method, ${ }^{14}$ are shown together in Fig. 6(a). The result clearly showed that the right edge of the line profiles shrank along a tilted line with an angle around $16^{\circ}$ from the perpendicular axis (Fig. 6(a)), as predicted in Fig. 3. The heights $H$, over the region the surface angle is sharper than the tilted line as defined in Fig. 3, were measured from all profiles. $H-A_{z}$ relationships (Fig. 6(b)), for both amplitude-decreasing and amplitude-increasing processes, showed a linear and reversible tendency. The slopes (a)

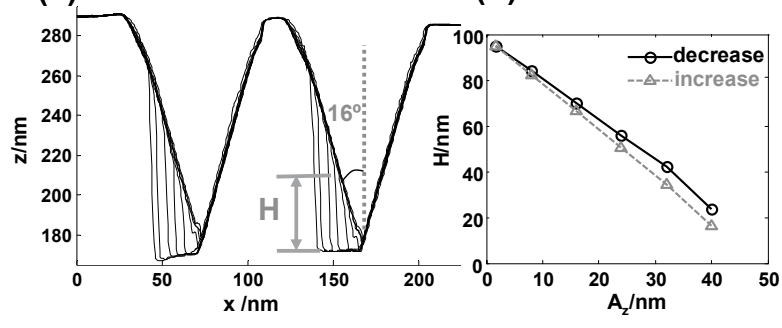

Fig. 6 (a) All experimental profiles after the alignment to remove the thermal drift, showing similar tendencies to those discussed in Fig. (3). The cantilever tilted angle was estimated as $16^{\circ}$ and $H$ is the height over the peak-contact region. (b) $\mathrm{H}-\mathrm{A}$ relationships for decreasing/increasing amplitude. The slopes were fitted around 1.7 and 1.9, respectively, close to the value 2 deduced in Eq. (5).

were fitted to be around 1.7 for decreasing curve and 1.9 for increasing curve, which are close to the prediction of slope 2 in Eq. (5). The error might come from the nonlinearity and calibration errors in $x / y / z$ direction. The results demonstrated the special amplitude effect in the newly established dynamic convolution model. And the measured $16^{\circ}$ is an estimation of the cantilever tilt angle, which is designed to be $12^{\circ}$ in the AFM used in the experiments. ${ }^{15}$

Therefore, the dynamic convolution model is of special significance to precisely and quantitatively measure the sharp nanostructures and will be especially useful in CD measurements ${ }^{16,17}$ and in the probe characterization..$^{7,18}$ For the critical dimension measurements in dynamic mode, the amplitude effect should be removed to get more accurate results. In the probe characterization, a perpendicular structure is generally used as the tip characterizer and a probe which can trigger amplitude effect such as what is shown in Fig. 2(c) will be characterized as a different $\left(P_{\mathrm{dt}}(x, y) \oplus P(x, y)\right)$, with an additional vibration factor included, from a general static $P(x, y)$. This will definitely lead to incorrect deconvolution: for the topography with the amplitude effect included to be deconvoluted, the deconvolution result is only correct in the case that the same amplitude as characterizing the probe is used; for the other dull specimen, $P_{\mathrm{dt}}(x, y)$ in the characterized probe is unwanted. The smaller imaging amplitude might help to obtain optimized information of a sharp structure. Sometimes, adjusting the direction of the specimen relative to the cantilever can also reduce the amplitude effect. For example, rotating the $2 \mathrm{D}$ specimen to a direction that the $2 \mathrm{D}$ extension direction is parallel to the long axis of the cantilever can minimize the amplitude effect for homogeneous structures.

Furthermore, the cantilever tilt has the impact not only on convolution but also on other measurements. The lateral vibration component, which results from the tilted vibration behavior, forces the probe to interact within a region with a lateral length of $(2 A \sin \alpha)$ instead of a fixed position, and so there is always the lateral "weighted average" effect in the measurements, which may affect various measurements. Based on this, a smaller amplitude indicates not only a focused interaction in $z$ direction but also a concentrated interaction in $\mathrm{x}$ direction. This might be one reason that high resolution image was obtained under small amplitude. ${ }^{19}$ 


\section{Conclusion}

In summary, we have established a novel convolution model suitable for dynamic mode by additionally convoluting a traditional contact convolution with an imaginary line probe, whose length equals to $2 A$ and direction the same as the vibration direction. The model indicates a strong amplitude expanded effect on the dynamic convoluted topography in the case that the contact convolution between the specimen and the probe has a place where the surface angle $\beta<\alpha-90^{\circ}$. The effect is especially significant for accurate and quantitative analysis such as in the probe characterization and in CD measurements in dynamic mode. Combined with other efforts in SPM, such as the standard samples and precise calibration methods under development recently, ${ }^{20}$ a more precise analysis will be realized.

\section{Acknowledgements}

This work was partially supported by the National Basic Research Program of China (Nos. 2006CB932505 and 2007CB936004), and by the Science and Technology Commission of Shanghai Municipality (1052nm07700).

\section{References}

1. C. Gerber and H. P. Lang, Nat. Nanotechnol., 2006, 1, 3.

2. R. Garcia, R. Magerle, and R. Perez, Nat. Mater., 2007, 6, 405.

3. D. Fujita, H. Itoh, S. Ichimura, and T. Kurosawa, Nanotechnology, 2007, 18, 084002.

4. D. Fujita, K. Onishi, and M. Xu, J. Phys.: Conf. Ser., 2009,
159, 012002

5. J. S. Villarrubia, J. Res. Nat. Inst. Stand. Technol., 1997, $102,425$.

6. C. Hahlweg, M. Gruhlke, and H. Rothe, Meas. Sci. Technol., 2009, 20, 084018.

7. H. Itoh, T. Fujimoto, and S. Ichimura, Rev. Sci. Instrum., 2006, 77, 103704.

8. L. O. Heim, M. Kappl, and H. J. Butt, Langmuir, 2004, 20 , 2760.

9. M. J. D'Amato, M. S. Marcus, M. A. Eriksson, and R. W. Carpick, Appl. Phys. Lett., 2004, 85, 4738.

10. R. J. Cannara, M. J. Brukman, and R. W. Carpick, Rev. Sci. Instrum., 2005, 76, 053706.

11. J. L. Hutter, Langmuir, 2005, 21, 2630.

12. S. A. Edwards, W. A. Ducker, and J. E. Sader, J. Appl. Phys., 2008, 103, 064513.

13. M. Stark, C. Moller, D. J. Muller, and R. Guckenberger, Biophys. J., 2001, 80, 3009.

14. J. P. Lewis, Vision Interface, 1995, 95, 120.

15. Practical Advice on the Determination of Cantilever Spring Constants, Veeco Instruments Inc., http://www.veeco.com/ pdfs/appnotes/AN94\%20Spring\%20Constant\%20Final_304. pdf.

16. K. Murayama, S. Gonda, H. Koyanagi, T. Terasawa, and S. Hosaka, Jpn. J. Appl. Phys., 2006, 45, 5928.

17. M. Yasutake, K. Watanabe, S. Wakiyama, and T. Yamaoka, Jpn. J. Appl. Phys., 2006, 45, 1970.

18. C. M. Wang, H. Itoh, J. L. Sun, J. Hu, D. H. Shen, and S. Ichimura, J. Nanosci. Nanotechnol., 2009, 9, 803.

19. S. V. Patil and P. M. Hoffmann, Adv. Eng. Mater., 2005, 7, 707.

20. I. Misumi, S. Gonda, O. Sato, K. Sugawara, K. Yoshizaki, T. Takatsuji, Y. Azuma, T. Fujimoto, and T. Kurosawa, Meas. Sci. Technol., 2007, 18, 2743. 